Bull. Mater. Sci., Vol. 3, Number 2, July 1981, pp. 209-216. C Printed in India.

\title{
Antiferromagnetism in the Heisenberg Hamiltonian
}

\author{
C K MAJUMDAR \\ Physics Department, University of Calcutta, 92, Acharya Prafulla Chandra Road, \\ Calcutta 700009, India
}

MS received 15 January 1981

\begin{abstract}
The shortcomings of the classical theories of antiferromagnetism are reviewed. On the basis of known exact results of several antiferromagnetic models, it is argued that the spin correlation functions may have oscillating behaviour anticipated from the classical Neel states even though the exact ground state is quite different from Neel state. It is also found that as the spin becomes large, the classical results become more and more applicable as seen in Fisher's model and verified experimentally. A few remarks on the broken symmetry are made. The existence of an anisotropic antiferromagnetic model with Neel states as ground state is pointed out.
\end{abstract}

Keywords. Antiferromagnetism; magnetic order.

\section{Introduction}

The understanding of antiferromagnetism in quantum mechanics is not as complete as that of ferromagnetism. The difficulty is that the structure of the ground state is rather complicated and usually cannot be explicitly written down for a given Hamiltonian. The theoretical situation is slightly better for the insulating salts than for metals. The familiar results due to Neel (1932), Van Vleck (1941), Anderson (1966) and others and quoted in text books (Kittel 1927) are fairly correct for the excitation spectrum for the large spin case and away from the transition point. The critical phenomena are now rather well explained by the renormalization group theories (Fisher 1974). We shall concentrate on the ground state, low-lying excited states, and the correlation functions in the Heisenbetg Hamiltonian.

Whether the isotropic Heisenberg Hamiltonian is a good description of real antiferromagnets will be discussed in the last section.

\section{Criticism of the usual results}

The Heisenberg Hamiltonian is

$$
\mathscr{H}=J \sum_{\langle i, j\rangle}^{\prime} \overrightarrow{S_{i}} \cdot \vec{S}_{j},
$$


where the sum goes over the sites $i$ and $j(i \neq j$ as indicated by the prime, the angular bracket \langle\rangle denotes that $i$ and $j$ are nearest neighbours). The classical spin wave theory is based on the sublattice picture (for a review, see Keffer 1966). In the simplest situation (that of bipartite lattices), there are two sublattices, and for each sublattice the Holstein-Primakoff transformation is used. The underlying ground state is assumed to be the Neel state-up spins on one sublattice and down spins on the other. Far from being the ground state the Neel states are not even eigenstates of the Hamiltonian (1). It can be shown that the ground state of (1) is non-degenerate, has total spin $s=0$ and has contributions from all the ${ }^{N} C_{N / 2}$ states of the $z$ component of total spin $s_{z}=0$.

The excited states have the energy dispersion relation

$$
\omega \sim k,
$$

for small wave vector $k$. The derivation does not reproduce the degeneracy correctly. Little is known about spin wave interaction in antiferromagnets.

The thermodynamic properties were established by Van Vleck in the mean free field approximation. The transition temperature is

$$
T_{c}=\frac{2}{3} J z S(S+1) / k_{B},
$$

where $z$ is the coordination number. Above $T_{e}$, the susceptibility satisfies the CurieWeiss law

$$
X=N g^{2} \beta^{2} S(S+1) /\left(3 k_{B}\left[T+T_{c}\right]\right),
$$

$\beta$ is the Böhr magneton and $g$ is the Lande factor. Below $T_{c}$ the substance shows spontaneous antiferromagnetism and one has to consider separately magnetic field parallel and perpendicular to the direction of sublattice magnetization. The perpendicular susceptibility $X_{\perp}$ is a constant equal to the value of $X$ at $T_{c}$

$$
X_{\perp}=N g^{2} \beta^{2} S(S+1) / 6 k_{B} T_{c},
$$

The parallel susceptibility $X_{11}$ is

$$
\begin{gathered}
\chi_{\| 1}=\frac{N g^{2} \beta^{2} S^{2} B_{S}^{\prime}\left(y_{0}\right)}{k_{B}\left[T+3 T_{c}(s+1)^{-1} S B_{S}^{\prime}\left(y_{0}\right)\right]}, \\
\text { where } y_{0}=2 z S J\left|\vec{S}_{0}\right| / k_{B} T,\left|\vec{S}_{0}\right|=S B_{S}\left(y_{0}\right) \cdot t
\end{gathered}
$$

$B_{S}(x)$ is the Brillouin function and the prime denotes its derivative. For $S=1 / 2$ equation (6) becomes

$$
\chi_{\mathrm{U}}=\frac{N g^{2} \beta^{2}}{4 k_{B}\left[T_{c}+\left(1-4 S_{0}{ }^{2}\right)^{-1} T\right]},
$$

while for the classical limit $S \rightarrow \infty, \beta \rightarrow 0$ the expression for $\chi_{11}$ is (with $\mu=g_{\beta} S$ ) 


$$
X_{11}=\frac{N \mu^{2}}{3 k_{B} T_{c}+k_{B} T\left[1-\left(S_{0} / S\right)^{2}-\frac{2}{8}\left(T / T_{c}\right)\right]^{-1}},
$$

The expression $4 S_{0}^{2}$ appearing in $(8)$ or $\left(S_{0} / S\right)^{2}$ in (9) has the interpretation of being the square of the ratio of the values of the spontaneous internal fields at the temperature $T$ and the absolute zero.

For a polycrystalline sample of a cubic crystal the susceptibility is

$$
X=\frac{1}{3} X_{11}+\frac{2}{3} X_{1}
$$

In summary, the susceptibility has a maximum and a cusp at $T=T_{c}$. The parallel susceptibility vanishes at $T=0$, while the perpendicular susceptibility remains large, thus creating a situation for spin flop transition.

It has been shown by Fisher (1960abc, 1962), that in antiferromagnets the thermodynamic transition occurs at a temperature $T_{N}$ lower than the temperature of the susceptibility maximum

$$
T_{N}<T\left(X_{\max }\right)
$$

and at $T_{N}$ the derivative of the parallel susceptibility diverges

$$
\partial X_{11} / \partial T \rightarrow \infty
$$

These results are explicitly seen in the exact solution of Fisher's model on antiferromagnet, which is derived from the Ising model.

Finally we come to the spin arrangements derived from the neutron scattering data. Thus in MnO spins in a single [111] plane are parallel, but in adjacent [111] planes they are antiparallel.

Recall that the neutron scattering is determined by correlation functions. The spin structures deduced are supposed to give the correct correlation functions. The question arises: what can be inferred about the ground state wavefunction from such data? Because the Neel states have the same up-down form as the spin structure deduced from the two sublattice model, an impression seems to persist that the wavefunction must have the structure of the spin structure. We show below that this need not be the case.

\section{The antiferromagnetic linear chain}

Many exact results are known in the spin-1/2 linear chain with isotropic exchange

$$
\mathscr{Z P}=2 J \sum_{i=1}^{N} \vec{S}_{i} \cdot \vec{S}_{i+1}
$$

$\left(J>0, N\right.$ even, $N+1 \equiv 1$ i.e. periodic boundary conditions, $\left.\left|\vec{S}_{i}\right|=1 / 2\right)$. The ground state has total spin $s=0$ and has energy -0.88629 NJ. The ground state is a combination of all the ${ }^{N} C_{N / 2}$ states with $s_{z}=0$. Its structure is not given explicitly but by the celebrated Bethe ansatz (Bethe 1931). Since the total spin is 0 , the average $\left\langle 0\left|S_{i}^{\mathrm{z}}\right| 0\right\rangle 0, \forall i$. The first excited states are $s=1$ states and the eigenvalues are given by 


$$
\hbar \omega=\pi J|\sin q|
$$

$q$ is the wave vector measured with respect to that of the ground state (the wave vector of the ground state is 0 if $N$ is a multiple of 4 and $\pi$ if $N$ is of the form $4 j+2$ with $j$ an integer). Here we have taken the lattice spacing to be unity. Notice the characteristic double periodicity of the spectrum (14).

The excitation spectrum from the spin wave theory is

$$
\hbar \omega=2 J|\sin k| \text {. }
$$

This differs from the exact result by a factor $2 / \pi$. However, the double periodicity of (15) is artificially produced, and the degeneracy of these states is not correct. Actually by numerical computation on short chains of higher spin particles it is possible to show that the spectrum (15) becomes correct in the limit $S \rightarrow \infty$, while the $S=1 / 2$ system is the extreme quantum limit (Jain et al 1975).

The correlation function $\left\langle S_{i}^{\mathrm{z}} S_{i+n}^{\mathrm{z}}\right\rangle$ in the ground state has not been calculated from the Bethe ansatz. Only the nearest neighbour correlation function $\left\langle S_{i}^{z} S_{i+1}^{z}\right\rangle$, which is connected to the ground state energy, is known and $\left\langle S_{i}^{z} S_{i+1}^{z}\right\rangle\left\langle 0\right.$. A detailed knowledge of the functions $\left\langle S_{i}^{z} S_{i+n}^{z}\right\rangle$ can be obtained from the short chain calculations (Bonner and Fisher 1964) (table 1). The long range order can be estimated:

$$
\lim _{n \rightarrow \infty}\left\langle S_{i}^{z} S_{i+n}^{z}\right\rangle=0 \text {, }
$$

The correlation function alternates in sign and falls off with the separation between the spins. Although the ground state is complicated, the correlation functions alternating in sign are reminiscent of the Neel states.

\section{Antiferromagnetic model with known ground state}

More about the relationship between the ground state wavefunction and the correlation functions can be learnt from the antiferromagnetic model studied by Majumdar and Ghosh (1969). Their Hamiltonian is

Table 1. Spin correlation functions of the linear chain as calculated by Bonner and Fisher 1964.

\begin{tabular}{cccccc}
\hline No. of spins & 4 & 6 & 8 & 10 & $\infty$ \\
\hline $4\left\langle S_{1}^{z} S_{2}^{z}\right\rangle$ & -0.667 & -0.623 & -0.609 & -0.682 & -0.591 \\
$4\left\langle S_{1}^{z} S_{3}^{z}\right\rangle$ & 0.333 & 0.277 & 0.261 & 0.254 & 0.25 \\
$4\left\langle S_{1}^{z} S_{4}^{z}\right\rangle$ & - & -0.309 & -0.252 & -0.231 & -0.19 \\
$4\left\langle S_{1}^{z} S_{5}^{z}\right\rangle$ & - & - & 0.199 & 0.173 & 0.15 \\
$4\left\langle S_{1}^{z} S_{6}^{z}\right\rangle$ & - & - & - & -0.188 & $\ldots$ \\
\hline
\end{tabular}




$$
\mathscr{H}=2 J \sum_{i=1}^{N} \vec{S}_{i} \cdot \vec{S}_{i+1}+J \sum_{i=1}^{N} \vec{S}_{i} \cdot \vec{S}_{i+2}
$$

$(J>0, N+1 \equiv 1, \mathrm{~N}+2 \equiv 2)$. The ground state energy is $E_{0}=-\frac{4}{8} \mathrm{NJ}$ and the ground state wave functions can be written as

$$
\begin{aligned}
\psi^{ \pm} & =[1,2][3,4] \ldots[N-1, N] \\
& \pm[2,3][4,5] \ldots[N-1],
\end{aligned}
$$

with $[l, m]$ representing a singlet pair

$$
[l, m]=\alpha(l) \beta(m)-\beta(l) \alpha(m)
$$

$\alpha$ and $\beta$ are the up and down spin eigenfunctions, respectively. If $T$ corresponds to unit translation, we have

$$
T \Psi^{+}=\Psi^{+}, T \Psi^{-}=-\Psi^{-} .
$$

The ground state has total spin zero, and $\left\langle 0\left|S_{i}^{z}\right| 0\right\rangle=0, \forall_{i}$. The order properties can be calculated exactly for the $N \rightarrow \infty$ limit and they show only short range order. If the evanescent order is studied for short chains, one gets a remarkable result (table 2): the corerrelation functions $\left\langle\sigma_{i}^{z} \sigma_{i+n}^{z}\right\rangle$ not only alternate in sign but settle down to a constant absolute value very much like what the Neel states suggest. The absolute value represents long range order which diminishes with increasing number of spins in the chain and ultimately disappears. Nevertheless, it suggests the view that the exact ground state wavefunction may have a quite complicated structure and yet show the alternating spin correlation functions expected from the Neel states. An observation of the Neel type spin correlation function does not say much about the exact ground states.

Table 2. Spin correlation functions for the state $\psi^{+}$(Similar results hold for $\psi^{-}$).

\begin{tabular}{cccccc}
\hline No. of spins & 6 & 8 & 10 & 12 & $\infty$ \\
\hline & & & & & \\
$\left\langle\sigma_{1}^{z} \sigma_{2}^{z}\right\rangle$ & -0.333 & -0.556 & -0.467 & -0.516 & -0.5 \\
$\left\langle\sigma_{1}^{z} \sigma_{3}^{z}\right\rangle$ & -0.333 & 0.111 & -0.067 & 0.030 & 0 \\
$\left\langle\sigma_{1}^{z} \sigma_{4}^{z}\right\rangle$ & 0.333 & -0.111 & 0.067 & -0.030 & 0 \\
$\left\langle\sigma_{1}^{z} \sigma_{5}^{z}\right\rangle$ & & 0.111 & -0.067 & 0.030 & 0 \\
$\left\langle\sigma_{1}^{z} \sigma_{6}^{z}\right\rangle$ & & & 0.067 & -0.030 & 0 \\
$\left\langle\sigma_{1}^{z} \sigma_{7}^{z}\right\rangle$ & & & & 0.030 & 0
\end{tabular}




\section{Fisher's classical $(S+\infty)$ model}

Fisher (1964) succeeded in evaluating the spin correlation function in the classical limit of large spin, $S \rightarrow \infty$.

Consider the Hamiltonian for $N+1$ atoms of spin $S$

$$
H=-2 J_{s} \sum_{i=0}^{N} \vec{S}_{i} \cdot \vec{S}_{i+1}-g_{s} \beta \vec{H} \cdot \sum_{i=0}^{N} \vec{S}_{i}
$$

The ground state energy for ferromagnetic case $\left(J_{0}>0\right)$.

$$
E_{0}=-2 J_{s} S^{2} N-(N+1) g, \beta H S .
$$

For taking the $S \rightarrow \infty$ limit, we now put

$$
2 J_{s} S^{2}=\frac{1}{2} J, g_{g} S=\frac{1}{2} g,
$$

and introduce the operators $\vec{s}_{j}=\vec{S}_{j} / S$. The commutation rules become

$$
s_{j}^{x} s_{j}^{y}-s_{j}^{y} s_{j}^{x}=\frac{1}{s} i s_{j}^{x},
$$

so that in limit $S \rightarrow \infty$, the operators $\vec{s}_{j}$ commute. The zerofield partition function and the spin correlation functions are for the antiferromagnetic case ( $J$ negative)

$$
Z_{N}=(\sinh K / K)^{N},
$$

and

$$
\left\langle S_{i}^{z} S_{i+1}^{x}\right\rangle=\frac{1}{3}(-1)^{|l|}[n(k)]^{\mid l i},
$$

$$
\text { where } u(k)=\operatorname{coth} k-\frac{1}{\mathrm{~K}}, K=\frac{1}{2}|J| / k_{B} T \text {. }
$$

The factor $(-1)^{11}$ is responsible for the oscillation. Experimental data (Hutchings et al 1972) on TMMC with $S=5 / 2 \mathrm{Mn}$-ion are well explained by the correlation functions. The spin $5 / 2$ and $7 / 2$ compounds already are fair approximations to the $S \rightarrow \infty$ limit. The quantum limit $S=1 / 2$, however, requires exact results such as (14), as seen in the experimental data on CPC (Endoh et al 1974).

\section{Broken symmetry}

The discussion of broken symmetry in antiferromagnets is due to Anderson (1964) but is not as rigorous as in the case of ferromagnets. Consider the solution (18). It is possible to construct a one-parameter family of ground states without translational symmetry. The symmetry broken is that of a discrete, infinite group, not a continuous group, so Goldstone's theorem cannot be directly applied in this case.

For the Heisenberg Hamiltonian (1), Anderson's argument is based on the two sublattice picture. Let $A$ and $B$ be the two sublattices, and $S_{A}$ and $S_{B}$ be, respectively, the sums of spins in these sublattices. $S_{A}$ and $S_{B}$ are rather like classical variables, and the lowest energy is obtained by setting $\left\langle S_{A}\right\rangle=-\left\langle S_{B}\right\rangle$, both being 
of order $N$, the number of spins present. In the exact state, of course, $\left\langle S_{A}\right\rangle=$ $\left\langle S_{B}\right\rangle=0$. Anderson suggests that this exact state is irrelevant, because there are many other states infinitesimally (order $1 / N$ ) close to it in energy. Thus if we take a state with $\left\langle S_{A}\right\rangle=-\left\langle S_{B}\right\rangle \sim N$, this will persist for a very long time but eventually will break up, as it is a wave packet composed of many states.

Anderson's description of the antiferromagnetic order must have the essence of physics in it. In this case the order parameter $S_{A}-S_{B}$ is not an exact constant of motion, but is approximately so. A rigorous mathematical discussion of this problem will have to take this fact into account.

\section{Anisotropy}

So far we have restricted our discussion to the isotropic antiferromagnetic exchange. It is, of course, known that all materials have anisotropy. Anderson's discussion of the broken symmetry shows that the picture of the sublattice magnetisation becomes more exact when anisotropy is present, because the system must tunnel rather than rotate its way around to the time-and spin-reversed state. It is also known that the Neel states are indeed the lowest states of the Ising model, an extremely anisotropic situation.

We may point out the existence of another Hamiltonian with the Neel states as ground states (Bose and Majumdar, unpublished),

$$
\mathscr{H}=2 J \sum_{+1}^{N}\left[S_{i}^{z} S_{i+1}^{z}+\Delta\left(S_{i}^{+} S_{i+1}^{+}+S_{i}^{-} S_{i+1}^{-}\right)\right] .
$$

Because the transverse anisotropy terms raise or lower nearest neighbours simultaneously, these terms have no effect on the Neel states for the spin $-1 / 2$ particles. The ground state energy can be shown to be $E_{0}=\frac{1}{2} \mathrm{JN}$ and it will have long rangeorder of the Neel states. A generalisation of this exists in three dimensions. Consider a bipartite lattice in three dimensions such that the nearest neighbours of the sublattice $A$ are on the sublattice $B$ and vice versa. Then the Hamiltonian

$$
\mathscr{H}=\underset{\substack{i \in \mathrm{A} \\ j \in \mathrm{B}}}{2 J} \sum_{i}\left[S_{i}^{z} S_{j}^{z}+\Delta\left(S^{+} S_{j}^{+}+S_{i}^{-} S_{j}^{-}\right)\right]
$$

(with only nearest neighbour coupling) again has Neel states as ground states and therefore has long range order in the ground state. The one-dimensional version (28) will not have long range order at finite temperatures, but (29) will probably have this property. A detailed analysis of the anisotropy and its role in the antiferromagnetic order should be illuminating.

It is a pleasure to dedicate this article to the memory of Professor A B Biswas who was deeply interested in the antiferromagnetic and ferrimagnetic materials.

\section{References}

Anderson P W 1964 Concepts in solids (New York: Benjamin) p. 175

Anderson P W 1966 Solid state physics eds F Seitz and D Turnbull (New York: Academic Press) 
Bethe H 1931 Z. Phys. 71205

Bonner J and Fisher M E 1964 Phys. Rev. 135 A140

Bose I and Majumdar C K (unpublished work)

Endoh Y, Shirane G, Birgeneau R J, Richards P M and Holt S L 1974 Phys. Rev. Lett. 32170

Fisher M E 1960a Physica 26618

Fisher M E 1960b Proc. R. Soc. (London) A 25466

Fisher M E 1960c Proc. R. Soc. (London) A256 502

Fisher M E 1962 Philos. Mag. 7173

Fisher M E 1964 Am. J. Phys. 32343

Fisher M E 1974 Rev. Mod. Phys. 46597

Hutchings M T, Shirane G, Biregeneau R J and Holt S L 1972 Phys. Rev. B5 1099

Jain C S, Krishan K, Majumdar C K and Mubayi V 1975 Phys. Rev. B12 5235

Kittel C 1977 Introduction to solid state physics 5th edition (New Delhi : Wiley Eastern) 479

Kittel C 1963 Quantum theory of solids (New York and London: John Wiley) Ch. 4 p. 49

Kefer F 1966 Handbuch der physik 181

Majumdar C K and Ghosh D K 1969 J. Math. Phys. 10 1388, 1399

Majumdar C K 1970 J. Phys. C3 911

Neel L 1932 Ann. Phys. 1764

Shastry B S and Majumdar C K 1973 Phys. Rev. B12 2856

Vleck J van 1941 J. Chem. Phys. 985 